\title{
Next generation therapies change the landscape in melanoma
}

\author{
Keith T. Flaherty
}

Address: Massachusetts General Hospital Cancer Center, 55 Fruit Street, Boston, MA 02114, USA

Email: kflaherty@partners.org

FI000 Medicine Reports 201 I, 3:8 (doi:10.3410/M3-8)

This is an open-access article distributed under the terms of the Creative Commons Attribution-Non Commercial License (http://creativecommons.org/licenses/by-nc/3.0/legalcode), which permits unrestricted use, distribution, and reproduction in any medium, provided the original work is properly cited. You may not use this work for commercial purposes.

The electronic version of this article is the complete one and can be found at: http://fl000.com/reports/m/3/8

\begin{abstract}
Melanoma is among the leading causes of years of life lost due to cancer. Current chemotherapy and cytokine-based immunotherapy approaches benefit only a small percentage of patients with advanced disease. However, the recent discovery of mutations in the gene encoding the serine-threonine kinase B-RAF (BRAF) raises the possibility that oncogene-targeted therapy may provide a new point of vulnerability. In parallel, a deeper understanding of the molecular mechanisms underlying antitumor T-cell activation and tolerance has provided a basis for developing therapies targeted against these processes. Results from an early phase trial with a BRAF inhibitor and a phase III trial with a novel agent that activates $T$ cells have radically altered the prospects for improving outcomes for patients with this historically treatment-refractory disease.
\end{abstract}

\section{Introduction}

Melanoma is one of the most devastating forms of cancer in terms of the number of years of life that patients lose, as it often affects younger people compared with other types of tumor. As with other cancers, chemotherapy has remained the mainstay of treatment for over half a century. The shortcomings of this approach are all too clear: when treated with chemotherapy, a diagnosis of metastatic melanoma generally meant a relentless progressive disease and death in approximately 6-9 months. Even immunotherapy, which has an accepted role in the treatment of metastatic melanoma patients, as well as those who have regional lymph node involvement that has been excised but remains at high risk of recurrence, fails to benefit more than a small minority of patients. Clearly, there is a need for a more effective treatment, and recently two more targeted approaches have begun to show great promise and hopefully will change the therapeutic landscape for melanoma. The first of these is the emergence of selective inhibitors of the serine-threonine protein kinase B-RAF (BRAF) for the treatment of metastatic melanoma, which have shown dramatic results in the $50 \%$ of patients whose tumors harbor an activating mutation in the oncogene encoding this kinase, $B R A F$, at least temporarily reversing the growth of the melanoma, resulting in a potential benefit in terms of life expectancy. Also, recent clinical trial results with novel immunologic therapies appear to be breaking the barriers observed with the previous generation of cytokine-based immunotherapies, and have produced some dramatic results. This review examines these developments in detail and discusses ways to improve treatment further.

\section{Oncogene-targeted therapy}

The identification of activating mutations in BRAF in $50-60 \%$ of melanomas in 2002 was the watershed event that opened the door for investigation of molecularly targeted therapy in melanoma [1]. BRAF is a constituent of the long-studied mitogen-activated protein (MAP) kinase pathway, a well-known mediator of growth factor signals in cancer. Therefore, when BRAF mutations were found in a large series of diverse tumor types, it was immediately apparent that these mutations might explain the involvement of this pathway in $7-8 \%$ of all cancers. As BRAF mutations are found most commonly in melanoma, laboratory investigation and clinical development of BRAF-targeted therapies naturally focused on this disease. The fundamental challenges that confronted the field were to understand why BRAF might be the tumors' Achilles 
heel rather than the other, numerous tumor-specific genetic alterations, and to develop potent and selective inhibitors.

For several years following the identification of BRAF mutations, sorafenib was the only targeted therapy in clinical development known to have some capacity for inhibiting RAF kinases. However, in phase II trials, sorafenib demonstrated only moderate MAP kinase pathway inhibition and little to no clinical efficacy as a single agent $[2,3]$. However, this moderate inhibition gave hope that more potent and selective BRAF inhibitors might yield better results, and consequently several BRAF inhibitors have entered clinical trials within the past few years. As PLX4032 and GSK2118436 are the first selective BRAF inhibitors to show an effect in patients, I will focus mainly on them.

In light of preclinical data demonstrating that PLX4032 had selective antitumor effects in tumors harboring $B R A F$ mutations, and because of the frequency with which $B R A F$ mutations are known to occur in melanoma, 49 out of the 55 patients with various types of tumor who were enrolled during the dose escalation phase of the small preliminary phase I clinical trial of PLX4032 had metastatic melanoma, and after the first evidence of measurable reductions in tumor size, the majority of patients enrolled had BRAF-mutant metastatic melanoma, which, rather unusually for a phase I trial, allowed not only for the characterization of safety and tolerability, but also efficacy [4]. It was apparent that the drug only worked in the BRAFmutant melanoma patients. As the dose was escalated to find the maximum tolerated dose, an increasing percentage of patients responded. By the time the highest five dose levels were evaluated, 11 of the 16 patients harboring the mutation experienced an objective response according to RECIST (Response Evaluation Criteria In Solid Tumors). All but two of these had at least some evidence of tumor regression. Dose-dependent toxicities that were commonly observed at the higher doses included rash fatigue, arthralgia, and photosensitivity. Severe rash, fatigue, and arthralgia ultimately dictated the maximum tolerated dose, but could be managed by interrupting therapy, when necessary, or with dose reduction.

Ultimately, 32 additional patients with BRAF-mutant metastatic melanoma were enrolled in the extension phase of the trial and given a dose of $960 \mathrm{mg}$ twice daily. A total of $81 \%$ of these patients showed measurable reduction in tumor size, two of these were tumor free, and four additional patients had minor degrees of tumor regression. Progression-free survival for these patients was generally greater than 7 months but it is too early to define overall survival. As a side effect, nearly one-third of these patients developed one or more skin lesionseither well-differentiated squamous cell carcinoma or keratoacanthoma. This finding is not so surprising: it has been known for many years that a subset of squamous cell carcinomas and keratoacanthomas harbor mutations in the gene encoding HRAS (a member of the RAS family of small GTPases), and more recently it has been observed that selective BRAF inhibitors induce MAP kinase pathway signaling, particularly in the setting of RAS mutations. It seems likely that these two factors interact to cause the appearance of these lesions during therapy. The clinical significance of squamous cell carcinomas and keratoacanthomas seems marginal in the setting of treatment for metastatic melanoma as they can be easily treated in most cases, but warrants more careful study.

The second BRAF inhibitor, GSK2118436, was evaluated in a phase I clinical trial [5] of similar design to the PLX4032 study, and the results were even more striking. GSK2118436 is even more potent in inhibiting BRAF than PLX4032 and is able to inhibit mutant BRAF kinase activity by $50 \%$ at concentrations less than $1 \mathrm{nM}$. A total of 93 patients were enrolled during the dose escalation portion of the trial, 85 of whom had metastatic melanoma, and of those, 76 had activating mutations in $B R A F$. Doses ranged from $12 \mathrm{mg}$ daily to $400 \mathrm{mg}$ daily. At the highest dose, dose-limiting toxicities were severe pyrexia and loss of consciousness. Among 35 patients treated at one of these two highest dose levels, the most common toxicities were pyrexia, fatigue, rash, the appearance of squamous cell carcinomas and keratoacanthomas, headache, nausea, and vomiting. Among the 16 patients treated at the two highest dose levels, there were 10 measurable responses observed (63\% response rate). GSK2118436 only failed to halt tumor growth progression in two patients treated at the higher dose levels at or before the first restaging evaluation. Of the nine patients who were enrolled with V600K mutations in BRAF, four had measurable responses. In addition to seeing responses in all visceral sites, treatment reduced the size of small, asymptomatic, previously untreated brain metastases in all of the subgroups of patients with this condition.

Taken together, the early clinical development of PLX4032 and GSK2118436 clearly confirm that BRAF inhibitors can (at least temporarily) halt or reverse disease in patients with melanomas carrying this mutation, apparently improving survival times compared with historically standard treatments (chemotherapy and interleukin-2), although the follow-up times are still too short to define median overall survival times. Even more dramatic results may be possible with further refinements. 


\section{Molecularly targeted immunotherapy}

Antitumor immune therapy has also taken a leap forward recently, following a breakthrough in understanding T-cell activation and anergy. Cytotoxic T-lymphocyte antigen 4 (CTLA4) is a T-cell surface receptor that competes with CD28 to bind with (T-cell stimulatory) B7 molecules on the surface of antigen presenting cells and promotes anergy. When the CD28:CTLA4 ratio is high, T-cell activation ensues, whereas anergy is the result of a low ratio. Thus an antibody targeting CTLA4 allows the B7/ CD28 interface to continue to drive T-cell activation, rather than become anergic, and this approach has been tested in the clinic in series of clinical trials spanning the past 10 years. The furthest advanced of this generation of therapies is ipilimumab, the CTLA4-blocking antibody that was recently shown to increase survival in metastatic melanoma patients in a (larger) phase III trial.

Although measurable responses were only seen in $10 \%$ of patients, or slightly less, proof-of-concept was established early on $[6,7]$ in small trials based on the durability of these responses. Some patients with advanced metastatic disease who had failed other therapies could be stabilized for months or even several years. However, a phase III trial was clearly needed to define the true size of the subpopulation that would benefit. In the first of these trials to be analyzed [8], patients with metastatic melanoma who had failed standard therapy such as dacarbazine were randomly assigned to receive ipilimumab in combination with a gp-100 peptide vaccine, ipilimumab alone, or peptide vaccine alone [8]. Because the gp-100 peptide can only be recognized by the human leukocyte antigen (HLA) marker HLA-A2, all patients were required to have this HLA type. Patients were randomly assigned such that $60 \%$ received ipilimumab/gp-100, 20\% received ipilimumab, and 20\% received the gp-100 vaccine alone. At the time of analysis, both of the ipilimumab-treated groups had a significantly improved survival compared to the control group. This difference was best shown when looking at longer follow-up time, such as two years after initiation of study therapy (24\% survival rate for ipilimumab alone versus $14 \%$ with vaccine alone).

There are two features of this approach that are unique compared to other standard cancer therapies. First, some patients receiving ipilimumab have radiographic evidence of disease progression during treatment, receive no subsequent therapy, and then manifest measurable responses months later. Amongst patients who demonstrate disease progression following the initial course of therapy (typically 3 months), there is a small percentage who will subsequently benefit. However, it is not currently possible to prospectively distinguish between patients who will experience unchecked progression and those with a delayed response to therapy. Second, ipilimumab induces activation not only of T cells that are capable of recognizing melanoma, but also of those recognizing self antigens, resulting in autoimmunity. A large percentage of patients develop some sort of mild or moderate autoimmunity in the first several months of therapy, requiring only minimal supportive care measures. The skin and gastrointestinal tract are the most common organs involved, manifesting as rash or diarrhea, but sometimes endocrine glands are involved. Severe diarrhea leading to dehydration and electrolyte deficiencies is a concern, but not as much as (rarer) gastrointestinal perforation. Amongst the endocrinopathies, the most clinically concerning is inflammation of the pituitary gland, which can lead to permanent adrenal insufficiency and hypothyroidism, and requires chronic mineral-corticoid and thyroid hormone replacement therapy. However, the life-prolonging benefit of this therapy is clear from the recently published randomized phase III trial, and outweighs these autoimmune side effects.

\section{Altered landscape}

Significant advances have been made in understanding the molecular pathophysiology, underlying genetic causes of tumors, and mediators of antitumor T-cell anergy. This has allowed the development of pharmacologic agents to specifically counter these processes. Even amongst patient populations with advanced, refractory melanomas, there is clear evidence of tumor regression and improved life expectancy with oncogene-inhibitors and T-cell stimulatory therapies. BRAF inhibition only works in those whose tumors harbor a BRAF mutation (approximately $50 \%$ of all melanomas), but induces responses in the vast majority of patients. Unfortunately, melanomas seem to develop resistance with prolonged treatment.

Ipilimumab, and other less-well-defined novel immunologic agents, are very effective in a small subgroup of patients, greatly extending life for these individuals, but as yet we don't understand the biological basis that predicts response. With this success as a basis to build on, it can be hoped that combinations of these and other approaches will confer more than temporary respite from metastatic melanoma in the future and provide long-term benefits to a higher proportion of patients.

\section{Abbreviations}

CTLA4, cytotoxic T-lymphocyte antigen 4; HLA, human leukocyte antigen; MAP, mitogen-activated protein. 


\section{Competing interests}

KTF has served as a consultant for Roche Pharmaceuticals, GlaxoSmithKline, and Bristol-Myers Squibb.

\section{References}

I. Davies H, Bignell GR, Cox C, Stephens P, Edkins S, Clegg S, Teague J, Woffendin H, Garnett MJ, Bottomley W, Davis N, Dicks E, Ewing R, Floyd Y, Gray K, Hall S, Hawes R, Hughes J, Kosmidou V, Menzies A, Mould C, Parker A, Stevens C, Watt S, Hooper S, Wilson R, Jayatilake H, Gusterson BA, Cooper C, Shipley J, et al:: Mutations of the BRAF gene in human cancer. Nature 2002, 41 7:949-54.

FI000 Factor 18

Evaluated by Channing Der 05 Jan 2002, Ulf Pettersson 09 Jul 2002, Kevan Shokat 22 Jul 2002, Alfred Wittinghofer 23 Jul 2002, Michael B Yaffe 06 Aug 2002

2. Eisen T, Ahmad T, Flaherty KT, Gore M, Kaye S, Marais R, Gibbens I, Hackett S, James M, Schuchter LM, Nathanson KL, Xia C, Simantov R, Schwartz B, Poulin-Costello M, O'Dwyer PJ, Ratain MJ: Sorafenib in advanced melanoma: a Phase II randomised discontinuation trial analysis. Br J Cancer 2006, 95:58I-6.

3. Flaherty KT, Redlinger M, Schuchter LM, Lathia CD, Weber BL, O'Dwyer PJ: Phase I/II, pharmacokinetic and pharmacodynamic trial of BAY 43-9006 alone in patients with metastatic melanoma. J Clin Oncol 2005, 23(Suppl):3037.

4. Flaherty KT, Puzanov I, Kim KB, Ribas A, McArthur GA, Sosman JA, O'Dwyer PJ, Lee RJ, Grippo JF, Nolop K, Chapman PB: Inhibition of mutated, activated BRAF in metastatic melanoma. $N$ Engl J Med 2010, 363:809-19.

FI000 Factor II

Evaluated by Barry Nelkin 29 Oct 2010, Mina Yaar 05 Nov 2010

5. Kefford RA, Arkenau H, Brown MP, Millward M, Infante JR, Long GV, Ouellet D, Curtis M, Lebowitz PF, Falchook GS: Phase I/II study of GSK2II8436, a selective inhibitor of oncogenic mutant BRAF kinase, in patients with metastatic melanoma and other solid tumors. J Clin Oncol 2010, 28(Suppl):8503.

6. Weber JS, O'Day S, Urba W, Powderly J, Nichol G, Yellin M, Snively J, Hersh E: Phase I/II study of ipilimumab for patients with metastatic melanoma. J Clin Oncol 2008, 26:5950-6.

7. Weber J, Thompson JA, Hamid O, Minor D, Amin A, Ron I, Ridolfi R, Assi H, Maraveyas A, Berman D, Siegel J, O'Day SJ: A randomized, double-blind, placebo-controlled, phase II study comparing the tolerability and efficacy of ipilimumab administered with or without prophylactic budesonide in patients with unresectable stage III or IV melanoma. Clin Cancer Res 2009. I 5:559|-8.

8. Hodi FS, O'Day SJ, McDermott DF, Weber RW, Sosman JA, Haanen JB, Gonzalez R, Robert C, Schadendorf D, Hassel JC, Akerley W, van den Eertwegh AJ, Lutzky J, Lorigan P, Vaubel JM, Linette GP, Hogg D, Ottensmeier CH, Lebbé C, Peschel C, Quirt I, Clark JI, Wolchok JD, Weber JS, Tian J, Yellin MJ, Nichol GM, Hoos A, Urba WJ: Improved survival with ipilimumab in patients with metastatic melanoma. N Engl J Med 2010, 363:7l I-23.

\section{Changes Clinical Practice}

FI000 Factor 8

Evaluated by Keith Flaherty 02 Aug 2010 